\title{
A Study on Teachers Perception about Components of English Handwriting in Pakistan
}

\author{
Hafiz Tahir Jameel* \\ Farhan Sarwar $^{* *}$ \\ Beverly H. Moskowitz ${ }^{* * *}$
}

\begin{abstract}
Handwriting is an essential element of school activities for the schoolaged children. Good or legible handwriting remains a constant task for the teachers and students throughout the whole day. Teachers recognize legible handwriting at a glance due to their routine work in the schools. Which criterion led to a teacher's determination of good handwriting was the purpose and research question posed in this study. The intent was to identify the components of legibility from the teachers' perspective. Referencing handwriting experts and a literature review, key variables were categorized and organized onto a 5-point Likert Scale questionnaire. Teachers' responses to the various legibility criteria were then tallied with regards to primary school students. Mean, standard deviation, exploratory factor analysis and path diagram statistics were applied to the ordinal data. It was concluded that twelve components were important for the legibility of handwriting of primary school students. These include Readability, Margin, Similarity, Line, Space, Size, Shape, Roundness, Form, Slant, Alignment and Recognition.
\end{abstract}

Keywords: assessment; language; quality writing; training

\footnotetext{
* Lecturer, Allama Iqbal Open University, Islamabad, Pakistan, Email: hafiztj@gmail.com

** Assistant Professor, University of Education, Lahore, Pakistan.

** President, Real OT Solutions, Educator and Occupational Therapist, US.
} 


\section{Introduction}

Even in the age of technology, manual transcription continues to be a vital and necessary life skill. Despite access to computers or other digital software and their ability to complete written or related assignments expeditiously, handwriting is a critical means to process information, express ideas and convey knowledge (Peverly, 2006). Additionally, since it is a low-tech option, manual writing survives because it is readily available, accessible and affordable. It is therefore not a surprising finding to discover the preponderance of occasions in which students are expected to write by hand (Faundez-Zanuy et al., 2020).

Handwriting and its associated tasks remained dominant activities in the primary schools. Manual writing continues to survive as a source of communication for students. Students spend most of the school time in handwriting and related activities. In fact, approximately 30 to $60 \%$ of schoolwork is comprised of handwriting or related activities (Cutler \& Graham, 2008; Volman, van Schendel, \& Jongmans, 2006). Children learn to write legible and meaningful words as part of their earliest formal education. Yet, even with the passage of time and successive grades, handwriting remains essential in the practical and academic life of the students (Khanam, 2020). Consequently, continued competence as manual writers enable students to have a convenient day-to-day vehicle to immediately transmit essential or informal notes. Achieving legibility, however, is a challenging and often elusive goal for teachers and therapists around the globe. This is due, in large part, to the fact that legible handwriting is a by-product of numerous variables.

Handwriting legibility is described as an ability of a human being to read handwritten individual characters on the basis of their appearance (Schneck, Amundson, Case-Smith, \& O'Brien, 2010). It is a broad term and is comprised of several components, including appropriate formation of words, adequate direction, suitable size, apposite tilt of letters, use of line, accurate use of the page, proper use of spacing between letters and words, and their location on the line (Feder \& Majnemer, 2007).

Academic achievement in schools is influenced by legibility of handwriting. Students with neat handwriting may be unknowingly considered smarter than their peers. Research confirms that students with legible handwriting earn higher grades than peers with illegible writing of similar context (Graham \& Harris, 2005). Accordingly, consistent legibility is both necessary for and predictive of academic success (Pollock et al., 2009).

Using a written sample, legibility may be assessed with regards to letter formation, slant, size and alignment (Pollock et al., 2009). Usually, 
two different methods are used to evaluate handwriting. The first is assessment of individual letters. The second is a global legibility assessment. It is the opinion of handwriting experts that assessment should focus on global legibility rather than formation of individual letters in a student's manuscript (Gregg \& Mather, 2002). Concentrating on global legibility avoids the use of laborious rating scales and provides a stronger estimate of overall readability of student's written work (Yakimishyn \& Magill-Evans, 2002). Because it can be used to assess functional handwriting of an entire classroom of students at the same time, it is considered a quick, simple, easy and efficient approach (Graham, Berninger, Weintraub, \& Schafer, 1998). Global legibility assessments address temporal components of writing too, since individual letters are expected to be better at the beginning of writing than in the middle or end. Similarly, legibility may be better in short versus long writing tasks, or copying over narrative writing (Graham et al., 1998). The inclusiveness of these general qualities, as opposed to specific criteria, in the full understanding of functional legibility are the basis for both generative and copied writing samples being characterized as legitimate components of a comprehensive global assessment (Rosenblum, Parush, \& Weiss, 2003).

There are several different measures of handwriting, each with its merits and limitations. The Test of Legible Handwriting (TOFH) is an example of a global assessment of handwriting recommended for grade 2 to 12 (Larsen \& Hammill, 1989). It has an average test-retest correlation of 0.9 with inter-rater reliability of 0.95 . Like many other global legibility assessment tools, it did not provide criterion reference information (Cizek, 2004; Graham, 1986).

Teacher's role is critical in the assessment of legibility of handwriting in a school setting (Sudsawad, Trombly, Henderson, \& Tickle-Degnen, 2001). Teacher's awareness about legibility components and their role in this type of assessment is essential in order to make accurate conclusions about legibility in that environment. It is especially urgent to understand and address deficits in the primary grade levels before maladaptive and illegible habits are formed.

The present study examined handwriting legibility in primary school students. The study was based on the essential components of legibility of handwriting as per teachers' perception about this concept. Determining the essential components of handwriting legibility among primary school students was the first objective of this study. Ascertaining the teachers' perception on essential components of legibility of handwriting was the second objective. This research work was designed 
to classify and categorize the teacher's perception about components of legibility of handwriting.

\section{Methodology}

A descriptive research designed using survey method was adopted for this research to investigate teachers' perception about components of English handwriting of students in primary schools. Survey design is a valuable scientific instrument. It is especially well-suited for noting opinions and for correlating opinions with respondent's sentiments (Kerlinger, 1984). Fourteen variables were identified as keys to measuring handwriting legibility among the target population. The teachers of the primary schools was requested to rate each variable for the said construct. Common essential variables were selected from the data provided on the basis of teachers' response about components of legibility of handwriting.

\section{Participants}

This included the teachers from the 56 primary schools from within the four main regions i.e. East, West, North and South of the district Faisalabad. Each region has approximately the same number of schools in its territory. A total fourteen schools were selected from each East and North region. Fifteen primary schools were taken from South region. The West region of the district was represented with thirteen primary schools. A sample of 388 teachers was selected randomly from the selected schools.

\section{Instrumentation and Scale Construction}

A number of handwriting assessors were available for the handwriting measurements. The handwriting evaluation tools vary according to their need and scope. The variation in the tools depended upon the need of the assessment. While the teachers' perceptions about legibility were the focus of the present study, there was little written about this in the literature. Fortunately, there was a consensus among these authors that primary school teachers were highly qualified to weigh in on the subject of legibility since the expectation for manual writing remained crucial to their daily activities. As such, they were felt to have relevant, comprehensive and realistic ideas regarding the distinction between legible and illegible handwriting. For that reason, a questionnaire entitled Legibility Questionnaire for English (LQE) was created reflective of teacher expertise and experience. The LQE is 
designed to record teacher responses regarding handwriting legibility of primary level students. The LQE contained different components of legibility of handwriting of primary school students. The construct to be measured was the teacher's perception of legibility in viewing the overall quality of a student's handwriting. The construct was based on definitions of legibility components for primary school students as discussed in the relevant literature. A preliminary pool of variables was generated for the initial scale. From that pool of variables, 16 items were selected as key components of legibility. For the purpose of retaining congruency with the content domain, selected items were approved by two independent reviewers proficient in scale development procedure (Ang \& Huan, 2006). Two items were rejected due to redundancy. Three items were revised and renamed. Finally, 14 items were selected for data collection.

\section{Handwriting Legibility Items}

The following variables were suggested for legibility of English handwriting.

1. Readability: This refers to the ease with which a reader can understand written text. The readability of a particular text depends on both the quality and legibility of written content.

2. Margin: It is the efficient use of side margins of a paper for symmetrical writing.

3. Similarity: Uniformity of shapes and sizes of the written characters should be consistent for legible handwriting.

4. Line: It is the ratio of a written character with reference to line. It plays an important role in the overall appearance, and thus the readability of handwriting.

5. Plan: This refers to the graphic layout of the written words. A clearly organized plan may increase the legibility of handwriting. The teachers were asked about the role of the planning in legibility.

6. Dimension: The letter parts are linked appropriately, and their relative measurement may suffer the legibility.

7. Space: This refers to the amount of space between letters in words and between words in sentences.

8. Form: This explains individual letter value or shape and includes comparisons of small letters and/or compressed tall or descending letters.

9. Alignment: This recognizes whether words are appropriate with reference of the lines.

10. Size: It refers to how big or small a student writes, as well as the overall size of the words relative to one another. 
11. Shape: It focuses on the whole word as per original figure of the word.

12. Roundedness: Sharp contours of letters or words that should be circular detract from the legibility of handwriting.

13. Slant: Some characters are oblique in its nature depending upon the context of the words.

14. Recognition: The words should be recognized by readers. Wellwritten words will be easily understood and will lead to a higher score on legibility criteria.

The Legibility Questionnaire for English (LQE) was designed as a close-ended questionnaire. It included the 14 items described above (1.1 Scale Construction). The LQE was developed to elicit data from primary teachers about their perceptions of the components of handwriting legibility for English writers. The research questions informed the questionnaire items to ensure that the items of questionnaire accurately reflected the variables. The questionnaire was limited to these 14 variables only regarding teachers' perception of legibility in English handwriting.

The teachers were asked to rate the role of each item in the legibility of handwriting of primary school students on a 5- Point Likert scale as follows: $1=$ Poor, $2=$ Fair, $3=$ Average, $4=$ Good and $5=$ Excellent

Table 1

Questionnaire for the Collection of Responses of the from the Teachers

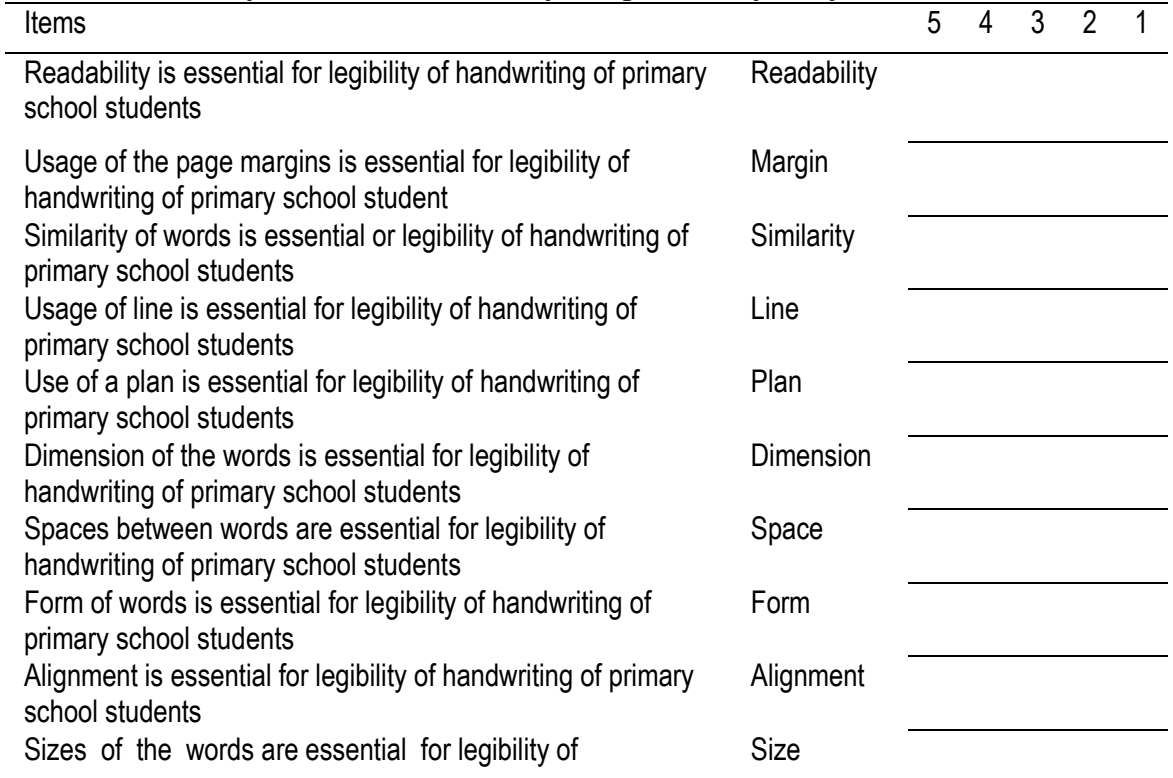


handwriting of primary school students

Shapes of the words are essential for legibility of handwriting of primary school students

Roundedness of words is essential for legibility of handwriting of primary school students

Slants of the words are essential for legibility of handwriting of primary school students

Words are easily recognizable

5 = Excellent, 4 = Good, $3=$ Average, 2 = Fair, $1=$ Poor

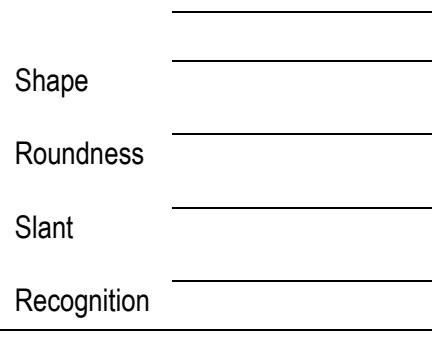

\section{Consent and Procedure}

The data was collected from a monthly meeting of the primary school teachers. Permission was granted by school principals for collection of data from their teachers. It was assured that the given data would be used for research purposes only. The purpose of the research was described to the teachers in advance of participation. The questionnaire was prepared and administered in English only. There was no need for translation since English was the language of instruction in the district where research was conducted. A copy of the questionnaire was presented to each participant. The teachers were asked to rate each response with reference to its importance in the legibility of primary school student's handwriting. The responses were graded in to a 5-point scale of increasing competence, progressing from poor to fair, average, good and then, excellent. Poor response represented the nil participation of the said variable in the legibility of handwriting. Excellent represented best compliance with the addressed components of handwriting legibility of the students. All questionnaires were completed in a single setting. Thirty minutes were allotted for the completion of the questionnaire. The first author assisted and remained available to the teachers as they responded to each item on the questionnaire, especially in case of question or clarification about the variables or questionnaire. The questionnaires were collected after 30 minutes. Distribution and collection of the questionnaire were assisted by three teachers.

\section{Data Analysis}

The Statistical Package of Social Sciences (SPSS) 16 and R 3.2.3 were used for the analysis of data. SPSS16 determined mean and standard deviation. R 3.23 was used for the factor analysis of the presented data. Mean scores were helpful in estimating average responses (Table 2). An exploratory factor analysis (EFA) was run to 
assess factor structure of scaled items. EFA was also used as a dimension reduction technique to explore interrelated structure among observed variables. EFAs are mostly used in studies to describe human intellectual abilities and are the suitable technique to categorize the groups (factors) in the data. The exploratory factor analysis model used was $\mathrm{Y}=\mathrm{X} \beta+\varepsilon$ where $\mathrm{Y}$ is a matrix of measured variables, $\mathrm{X}$ is a matrix of common factors, $\beta$ is a matrix of factor loadings, $\varepsilon_{\text {in }}$ is a matrix of unique factors. Communality is the variance of observed variables accounted for by a common factor. A large value of communality indicates strong contribution in that construct, and is a calculated by summing squares of factor loadings (Child, 2006).

In order to check the appropriate application of exploratory factor analysis on the data set, two tests were used. These include the Bartlett Test of Sphericity and Kaiser-Mayer Olkin Index. The former test compared the possibility of redundancy between the variables that could instead be summarized with fewer factors. In this method and under the null hypothesis of orthogonality of variables, the determinant of the sample correlation matrix of the variables is nearly one. It is the only factor needed if the variables are perfectly correlated. PCA and EFA are possible with the rejection of the above null hypothesis. The test statistics of Bartlett's test is chi square.

The Kaiser-Mayer Olkin Index was used to check whether the variables, with the concept of partial correlations, could be factorized efficiently or not. Factor analysis is useful when the value of KMO index is high (nearly 1) (Friel, 2007). Two selection criteria were adopted to assist in deciding the number of components to be retained. Only those factors having eigen values greater than 1 and a suggested scree plot were included (Cattell, 1988; Kaiser, 1960). Minimum residual (Minres) method was used for factor analysis. For goodness of fit or confirmation of selected factors, discrete cutoff criteria were presented in situations other than the usual Chi square goodness of fit and AIC, BIC criteria. For maximum likelihood-based Tucker Lewis Index, the cutoff value was0.95 and is 0.06 for RMSEA (Hu \& Bentler, 1999). Exploratory factor analysis (EFA) was performed by using minimum residual (Minres) method. Minres is considered suitable to minimize the offdiagonal residual correlation (Cramer, 2003) so that they might be treated as a single variable. The factor analysis culminated in two conceptual factors to accept and reject the variables for LQE. To make the structure more presentable, a path diagram was used to show the components of two factors along with their weight toward related factor (Revelle, 2015). 


\section{Results and Discussion}

The mean scores for each variable were as follows: Readability $=$ 3.56 , Margin $=2.53$, Similarity $=3.45$, Line $=3.36$, Space $=3.63$, Size $=$ 3.33, Shape $=3.34$, Roundedness $=2.92$, Form $=3.34$, Slant $=3.33$, Alignment $=3.39$ and Recognition $=3.38$. Plan and Dimension represented with mean value 2.23 and 2.25 (Table 2), respectively.

Before conducting EFA, suitability of the data was ensured by examining the results of two indicators. Kaiser-Mayer-Olkin test of sampling adequacy index was 0.91 and Bartlett's test of spherical was $\chi^{2}(91$, $\mathrm{N}=388)=3010.3, \mathrm{p}<0.001$ showed that both (sample and correlation matrix) were suitable to conduct EFA. The Principal component analysis (PCA) was performed on the obtained score from 14 items of LQE. Decisions about the number of factors were taken by the researcher using a combination of methods including scree plot, eigenvalue $>1.0$, parallel analysis along with conceptual clarity, simple structure and interpretability (Pollock et al., 2009). Two factors fulfilled the requirement of parallel analysis, eigenvalue $>1.0$, scree plot along with conceptual clarity and interpretability in this research. Formation of two factors was our goal in this study. P Placement of each item was automatically done by EFA. Two factors were shown in path diagram named rejected and accepted items. In addition, some items were weighted more than 0.4 for their related factor and others were weighted less than 0.4 for other factors (Stevens, 2012). Each item in this study was presented with more than 0.5 weight on its relevant factor and less than 0.01 eight on its opposite factor (Table 2).

Table 2

Pattern Matrices for the Legibility, Communalities, Means, and Standard Deviations

\begin{tabular}{lccccc}
\hline Items & Factor I & Factor II & $\mathrm{h}^{2}$ & $\mathrm{M}$ & $\mathrm{SD}$ \\
\hline Readability & 0.01 & 0.82 & 69.6 & 3.56 & 0.76 \\
Margin & 0.05 & 0.43 & 22.6 & 2.53 & 0.79 \\
Similarity & 0.00 & 0.80 & 76.6 & 3.45 & 0.72 \\
Line & -0.05 & 0.75 & 62.0 & 3.36 & 0.80 \\
Plan & 0.90 & 0.00 & 89.4 & 2.23 & 0.91 \\
Dimension & 0.79 & 0.01 & 88.6 & 2.25 & 0.93 \\
Space & 0.01 & 0.52 & 4.9 & 3.63 & 0.83 \\
Size & 0.00 & 0.79 & 66.3 & 3.33 & 0.71 \\
Shape & -0.03 & 0.82 & 70.5 & 3.34 & 0.71 \\
Roundedness & 0.01 & 0.78 & 64.9 & 3.34 & 0.70 \\
Form & 0.04 & 0.57 & 0.0 & 2.92 & 0.91 \\
Slant & 0.01 & 0.77 & 64.1 & 3.33 & 0.67 \\
Alignment & 0.05 & 0.76 & 63.2 & $3 . .39$ & 0.65 \\
Recognition & 0.05 & 0.82 & 70.3 & 3.38 & 0.79 \\
\hline Note: $M=$ Mean, SD & Standard Deviation, $\mathrm{h}^{2}=$ Communalities & &
\end{tabular}


There is no need of item reduction. Two items were recruited for factor one for the accepted components and the other factor for rejected components by sung EFA and Path diagram.

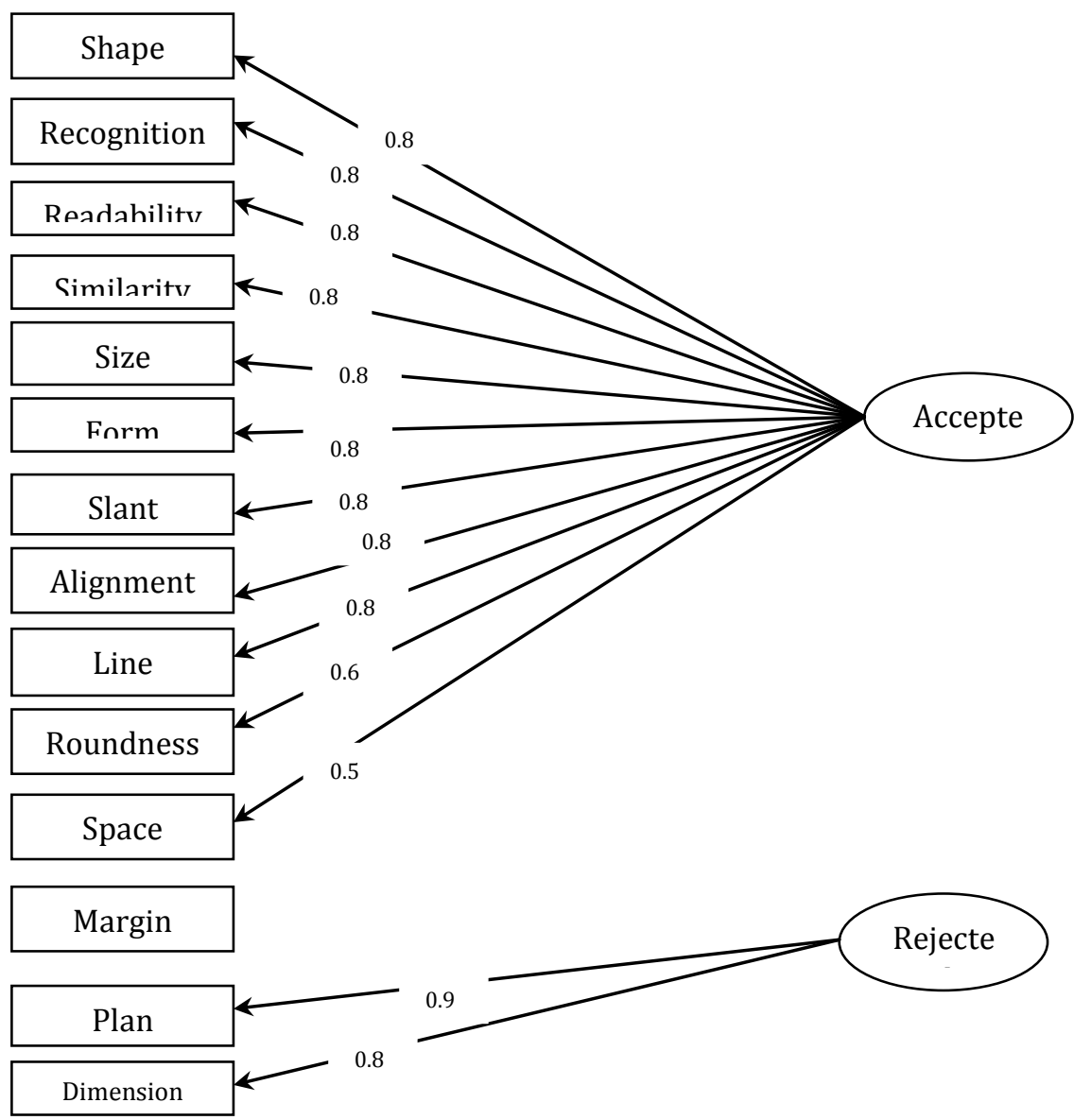

Structure coefficients of factors along with mean, standard deviation and communalities of measured variables are represented in Table 1. All items presented with communalities $\left(h^{2}\right)$ of 1.0. Rejected component had two items (e.g. Plan and Dimension) and was considered as not participating in the legibility as explained by $44.62 \%$ of cumulative variance. Accepted component contained twelve items, namely Readability, Margin, Similarity, Line Space, Size, Shape, Roundedness, Form, Slant, Align and Recognition. These variables had $57.42 \%$ of cumulative variance and were considered important determinants for handwriting legibility of students as per teacher's perception (Uchimura, Muraoka, \& Ikeda, 2019). The present study 
therefore proposed 12 components as indicators of legibility of students' handwriting. The quality of handwriting was assessed with the help of ten variables from a previous study (Rosenblum, 2008).

Many questionnaires were used to measure handwriting. These questionnaires measured different aspects of handwriting along with its legibility. Evaluation Tool of Children's Handwriting Manuscript (ETCH-M) is an example of such a tool. It is used to measure handwriting legibility of words and numbers. The presented questionnaire, the LQE, was specifically designed to assess handwriting legibility (Yu \& Chang, 2019). Three components of LQE i.e. slant, spacing and letter sizes were also represented in the ETCH-M, and were considered as essential variables of legibility (Diekema, Deitz, \& Amundson, 1998). Slant, spacing and letter sizes were likewise valued as important variables for the legibility of handwriting.

\section{Internal Consistency}

Internal consistency was measured from the presented data, also. Estimation of internal consistency was made by using Cronbach's coefficient alpha. It was measured on thee level. First, the internal consistency of the 14 variables was measured in combination. Then, internal consistency was calculated in individually. Next, internal consistency of 12 accepted components were measured. Lastly, the internal consistency of 2 rejected variables was measured. Obtained score from 14 items of LQE had Cronbach's alpha of 0.81 . Internal consistency estimates for two factors were given: Factor 1 (MR 1) of 402 (two; $\alpha=0.88$ ) and MR 2 (twelve; $\alpha=0.86$ ). The presented Cronbach's alpha estimates seem to be adequate for common research purposes. This is in line with previous research findings (Graham, Struck, Santoro, \& Berninger, 2006; Uchimura et. al.,).

\section{Conclusions}

Measurement of legibility involves assessment of numerous qualities of handwriting. The researchers adopted different ways for the assessment of legibility as per the inclusiveness and protocol of the assessment. Identification of essential components of legibility helps teachers satisfactorily assess their students' handwriting. The implications for daily usage in schools were significant. For this research, components of legibility were identified and weighted according to the teacher's perception. In pursuit of these variables, the 
proposed research question might be worded as, "What are the legibility components of English handwriting as per teacher's perceptions?"

This study provided empirical evidences to show that the variables primary teachers considered most contributory toward legibility for the primary school students include Readability, Margin, Similarity, Line, Space, Size, Shape, Roundedness, Form, Slant, Align and Recognition. Addressing these variables may be helpful when pursuing handwriting improvement among primary school students throughout a routine school day. Plan and Dimension variables were not seen as important for legibility of handwriting according to the perception by teachers of primary level students.

In conclusion, this study was based on the teachers' perceptions on the legibility of handwriting. The legibility of handwriting of the primary school students may be assessed on the basis of the 12 variables identified by primary teachers and mentioned in this research work. The improvement of these components may have accumulative effect in the legibility of handwriting for the students of primary schools. Attention toward these 12 factors may be helpful in promoting a positive teachers' attitude regarding legible handwriting. Additionally, overall legibility may be assessed by schoolteachers through their understanding and examination of these variables. Therefore, from the findings of this study, it can be stated with confidence that all these components affect the legibility of handwriting.

\section{Recommendations}

Based on the findings of this study, the researchers make the following recommendations:

- Teachers should address the readability, margin, similar, line, space, size, shape, roundedness, slant, alignment and recognition when teaching and remediating legibility of English handwriting of students in the primary schools.

- Teachers may use appropriate techniques for the improvement of readability, margin, similarity, line, space, size, shape, roundedness, slant, alignment and recognition for the improvement of legibility of English handwriting among primary school students.

- $\quad$ The scale may be used for further exploration by using suitable techniques (e.g. exploratory factor analysis) with more conceptual clarity. 


\section{Declaration of Conflict of Interest}

Authors declare that there is no conflict of interest.

\section{Acknowledgement}

We are thankful to Dr. Muhammad Yaseen for his assistance and guidance in the statistical analysis of the data. We are also grateful to the school teachers who participated in the study.

\section{Funding}

The researchers did not receive any financial support for the implementation of the research nor the authorship of this article. 


\section{References}

Ang, R. P., \& Huan, V. S. (2006). Academic expectations stress inventory development, factor analysis, reliability, and validity. Educational and Psychological Measurement, 66(3), 522-539.

Cattell, R. B. (1988). The meaning and strategic use of factor analysis. In Handbook of multivariate experimental psychology (pp. 131-203): Springer.

Child, D. (2006). The essentials of factor analysis: A\&C Black.

Cizek, G. (2004). Review of the Test of Legible Handwriting. Mental Measurements Yearbook, 11.

Cramer, D. (2003). Advanced quantitative data analysis: McGraw-Hill Education (UK).

Cutler, L., \& Graham, S. (2008). Primary grade writing instruction: A national survey. Journal of Educational Psychology, 100(4), 907-919.

Diekema, S. M., Deitz, J., \& Amundson, S. J. (1998). Test-retest reliability of the evaluation tool of children's handwritingmanuscript. American Journal of Occupational Therapy, 52(4), 248255 .

Faundez-Zanuy, M., Fierrez, J., Ferrer, M. A., Diaz, M., Tolosana, R., \& Plamondon, R. (2020). Handwriting biometrics: Applications and future trends in e-security and e-health. Cognitive Computation, 12(5), 940-953.

Feder, K. P., \& Majnemer, A. (2007). Handwriting development, competency, and intervention. Developmental Medicine \& Child Neurology, 49(4), 312-317.

Friel, C. M. (2007). Notes on factor analysis. Criminal Justice Center, Sam Houston State University.

Graham, S. (1986). A review of handwriting scales and factors that contribute to variability in handwriting scores. Journal of School Psychology, 24(1), 63-71.

Graham, S., Berninger, V., Weintraub, N., \& Schafer, W. (1998). Development of handwriting speed and legibility in grades 1-9. The Journal of Educational Research, 92(1), 42-52. 
Graham, S., \& Harris, K. R. (2005). Improving the writing performance of young struggling writers theoretical and programmatic research from the center on accelerating student learning. The journal of Special Education, 39(1), 19-33.

Graham, S., Struck, M., Santoro, J., \& Berninger, V. W. (2006). Dimensions of good and poor handwriting legibility in first and second graders: Motor programs, visual-spatial arrangement, and letter formation parameter setting. Developmental Neuropsychology, 29(1), 43-60.

Gregg, N., \& Mather, N. (2002). School Is Fun at Recess Informal Analyses of Written Language for Students with Learning Disabilities. Journal of Learning Disabilities, 35(1), 7-22.

Hu, L. t., \& Bentler, P. M. (1999). Cutoff criteria for fit indexes in covariance structure analysis: Conventional criteria versus new alternatives. Structural equation modeling: a multidisciplinary journal, 6(1), 1-55.

Kaiser, H. F. (1960). The application of electronic computers to factor analysis. Educational and Psychological Measurement.

Kerlinger, F. N. (1984). Liberalism and conservatism: The nature and structure of social attitudes: Lawrence Erlbaum Assoc Incorporated.

Khanam, M. (2020). Scientific Handwriting Changes Your Life. International Journal of Research in Engineering, Science and Management, 3(11), 13-15.

Larsen, S., \& Hammill, D. (1989). Test of legible handwriting. Austin, TX: Pro-Ed.

Peverly, S. T. (2006). The importance of handwriting speed in adult writing. Developmental Neuropsychology, 29(1), 197-216.

Pollock, N., Lockhart, J., Blowes, B., Semple, K., Webster, M., \& Farhat, L. (2009). Handwriting assessment protocol. In: Hamilton: CanChild Centre for Childhood Disability Research, School of Rehabilitation Science, McMaster University.

Revelle, W. (2015). An overview of the psych package.

Rosenblum, S. (2008). Development, reliability, and validity of the Handwriting Proficiency Screening Questionnaire (HPSQ). American Journal of Occupational Therapy, 62(3), 298-307. 
Rosenblum, S., Parush, S., \& Weiss, P. L. (2003). Computerized temporal handwriting characteristics of proficient and non-proficient handwriters. American Journal of Occupational Therapy, 57(2), 129-138.

Schneck, C., Amundson, S., Case-Smith, J., \& O'Brien, J. (2010). Prewriting and handwriting skills. Occupational Therapy for Children, 6, 555-580.

Stevens, J. P. (2012). Applied multivariate statistics for the social sciences: Routledge.

Sudsawad, P., Trombly, C. A., Henderson, A., \& Tickle-Degnen, L. (2001). The relationship between the Evaluation Tool of Children's Handwriting and teachers' perceptions of handwriting legibility. American Journal of Occupational Therapy, 55(5), 518-523.

Uchimura, S., Muraoka, T., \& Ikeda, H. (2019). A Method for Handwriting Analysis of Signature Using Mahalanobis Distance. Paper presented at the 2019 IEEE 8th Global Conference on Consumer Electronics (GCCE).

Volman, M., van Schendel, B. M., \& Jongmans, M. J. (2006). Handwriting difficulties in primary school children: A search for underlying mechanisms. American Journal of Occupational Therapy, 60(4), 451-460.

Yakimishyn, J. E., \& Magill-Evans, J. (2002). Comparisons among tools, surface orientation, and pencil grasp for children 23 months of age. American Journal of Occupational Therapy, 56(5), 564-572.

Yu, N.-Y., \& Chang, S.-H. (2019). Characterization of the fine motor problems in patients with cognitive dysfunction-A computerized handwriting analysis. Human Movement Science, 65, 71-79.

\section{Citation of this Article:}

Jameel, H.T., Sarwar, F., \& Moskowitz, B. H. (2020). A study on teachers' perception about components of English handwriting in Pakistan. Pakistan Journal of Education, 37 (2), 97-112. 